\title{
Inflammation: A Contributor to Depressive Comorbidity in Inflammatory Skin Disease
}

\author{
Delaram Farzanfar ${ }^{a}$ Yekta Dowlati ${ }^{b}$ Lars E. French ${ }^{c}$ Michelle A. Lowes ${ }^{d}$ \\ Afsaneh Alavi $^{\mathrm{e}}$ \\ a University Health Network, University of Toronto, Toronto, ON, Canada; ${ }^{b}$ Centre for Addiction and Mental Health, \\ Toronto, ON, Canada; ' Zurich Hospital University, Zurich, Switzerland; d The Rockefeller University, New York, NY, \\ USA; ${ }^{e}$ Department of Medicine, University of Toronto, Women's College Hospital, Toronto, ON, Canada
}

\section{Keywords}

Inflammation - Depression · Skin disorders · Psoriasis .

Atopic dermatitis $\cdot$ Hidradenitis suppurativa

\begin{abstract}
The prevalence of affective disorders such as depression and anxiety is particularly high in patients with autoimmune diseases, including inflammatory skin diseases such as psoriasis, atopic dermatitis, and hidradenitis suppurativa. A dysregulated immune response has been linked to the precipitation of depression in many patient populations. However, studies examining the extent to which the underlying skin disease inflammatory processes contribute to depression and a subsequent decline in quality of life are limited. The published literature over the past 5 years was reviewed for evidence of a relationship between depression and inflammatory processes in the context of skin pathology. The findings, particularly the evidence from interventional clinical trials of targeted anti-cytokine therapies, suggest that proinflammatory cytokines associated with several skin diseases may be causally linked with the coexistent depressive symptomology.

(c) 2018 S. Karger AG, Basel
\end{abstract}

\section{Introduction}

The prevalence of affective disorders in dermatological patients is high [1]. Approximately $30 \%$ of patients with skin disorders suffer from a comorbid psychological condition [2,3], such as depression, anxiety, and somatization [1]. Chronic inflammatory skin diseases such as psoriasis (PS), atopic dermatitis (AD), and hidradenitis suppurativa (HS) are particularly debilitating skin disorders which have a heavy psychological burden [4-6]. The disability reported in cases of PS is comparable to illnesses such as cancer and coronary artery disease [7].

Depression is reported in up to $42 \%$ of patients with HS in the USA, and in a large study involving 3,207 patients, there was an odds ratio of 1.7 for depression in people living with HS compared to other dermatological patients $[8,9]$. In another study on 154 patients with HS, depressive symptoms were reported in more than $70 \%$ of the cases; 55 patients $(34.7 \%)$ had depression and 32 (20.8\%) had borderline depressive symptoms [10].

Patients with $\mathrm{AD}$ and $\mathrm{HS}$ experience high suicidality according to several studies $[11,12]$. However, it should be noted that a recent systematic review on the risk of sui-

\section{KARGER}

(c) 2018 S. Karger AG, Basel

E-Mail karger@karger.com

www.karger.com/spp
Afsaneh Alavi, MD, MSc, FRCPC

Women's College Hospital, University of Toronto

76 Grenville St, 5th Floor

Toronto, ON M5S 1B2 (Canada)

E-Mail afsaneh.alavi@utoronto.ca 


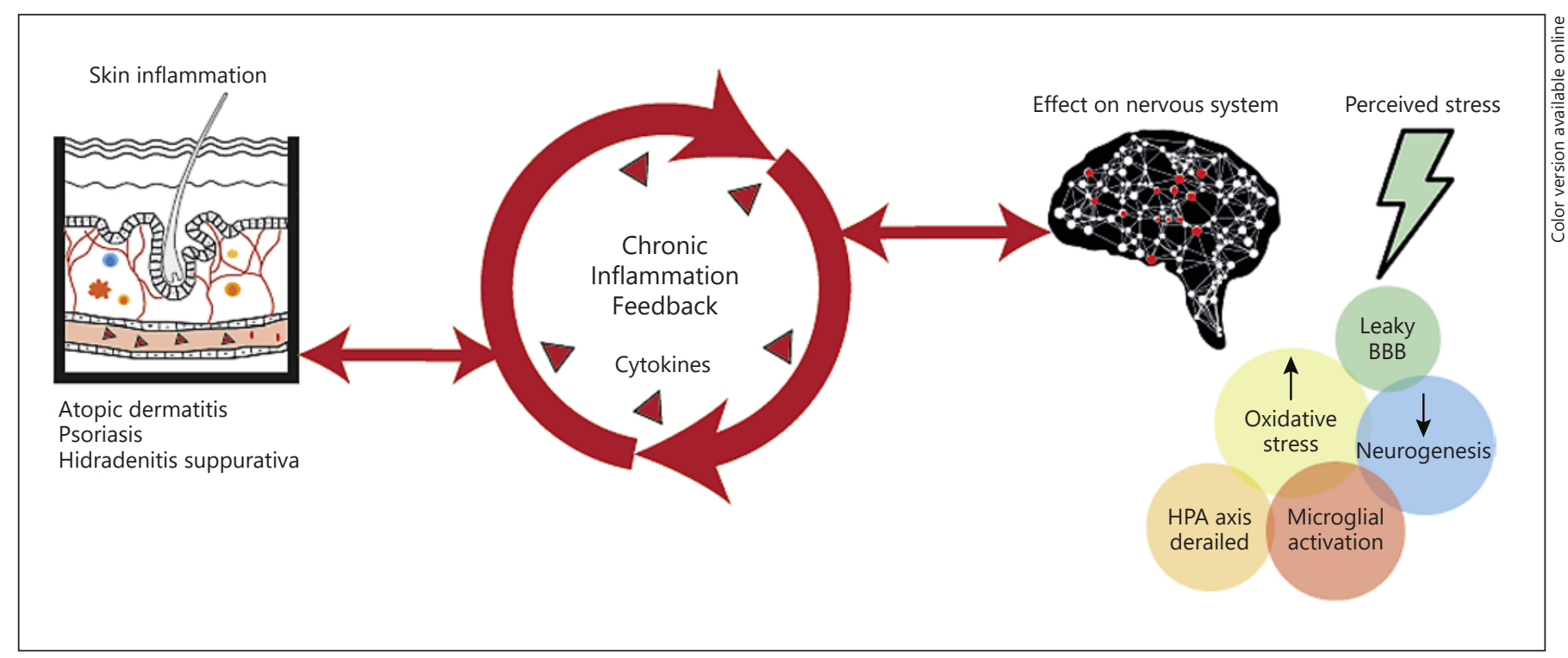

Fig. 1. The potential relationships between cutaneous inflammation and central nervous system contributions form a chronic feedback loop.

cidality in PS patients found no increased risk in suicide among these patients [13]; the low methodological quality for the included studies warrants further research. Huilaja et al. [14] reported the psychological burden of HS in a large Finnish study, with at least 1 psychiatric disorder in $24 \%$ of HS patients, compared to $19 \%$ of the patients with PS and $13.5 \%$ with dysplastic nevus.

It is difficult to decipher the extent to which depressive symptoms present in patients with these dermatological disorders is due to the underlying cutaneous inflammatory disease compared to the psychosocial impact of living with chronic illness. The disfiguring and visible nature of chronic inflammatory skin diseases undoubtedly contributes to their psychosocial impact; however, the prevalence of depression in PS, for example, is higher than similarly disfiguring skin disorders such as ichthyosis [4]. The aim of this study is to review the recent literature for the evidence of a relationship between depression and the underlying inflammatory processes characterized in PS, AD, and HS [4]. These proposed relationships are outlined in Figure 1.

\section{Materials and Methods}

A search of the PubMed, PsycINFO, and Cochrane databases was conducted for any relevant English articles published from 2012 to 2017, using the following terms: (inflammation OR inflammatory OR cytokine) AND (depression OR mood OR psych*)
AND (skin OR derm*) AND (atopic dermatitis OR hidradenitis suppurativa OR psoriasis). Moreover, reference lists of any review, meta-analysis and related articles as well as clinical trial registries were searched for any missing published and unpublished studies. There were 240 records from this search which were combined with hand searches, to result in 273 entries. Duplicates, non-English articles, and animal studies were excluded. A breakdown of the total search for each disease included 46,112 , and 16 records for $\mathrm{AD}, \mathrm{PS}$, and HS, respectively. The relevant abstracts were reviewed; 42 papers were selected for full-text review and included in the manuscript.

\section{Discussion}

Potential Mechanisms for a Pro-Inflammatory State Contributing to Psychological Effects

There are a number of important mechanisms through which a pro-inflammatory state may contribute to depression, including the psychological effects of circulating mediators and direct effects on the microglia.

There are considerable data showing that elevated levels of circulating cytokines can have a psychological impact. Cytokines such as interleukin (IL)-6 and tumor necrosis factor (TNF)- $\alpha$ can be detected in the serum during major depression, and they exhibit a dose-response relationship with the severity of depression $[15,16]$. In studies where interferon immunotherapy was used for the treatment of viral hepatitis and cancer, increased psycho- 
logical distress including depression was provoked in patients, even in those without a psychiatric history $[17,18]$. Furthermore, the activation of the immune system has been shown to induce mood changes which resemble sickness behaviors and overlap with the behavioral symptoms of depression such as anhedonia, anorexia, and social withdrawal [19]. Reichenberg et al. [20] investigated the effects of low-dose endotoxemia on emotional and cognitive function, and found that following an endotoxin injection, there is a transient increase in the levels of anxiety and depressive symptoms and a decrease in memory performance, measured using the state anxiety inventory, the depression adjectives check list, and a battery of neuropsychological tests, respectively.

Although inflammation may be local, arising in peripheral organs, pro-inflammatory cytokines such as TNF- $\alpha$ and IL-6, but also dendritic cells, can cross the blood-brain barrier (BBB) and trigger a cascade of events in the central nervous system [21]. The integrity of the $\mathrm{BBB}$ can be compromised due to stress, leading to a "leaky BBB" which does not adequately protect the brain from peripheral cytokine infiltration [22]. An overactive stress response [23] and elevated levels of peripheral cytokines can lead to brain cytokine signaling with downstream effects on synaptic function via the actions of the microglia, neurotransmitter metabolism, and neurogenesis [22]. For example, increased levels of TNF- $\alpha$ accelerate the breakdown of neurotransmitters, such as serotonin, by increasing the activity of the enzyme indoleamine 2,3-dioxygenase [24]. Furthermore, central inflammation has also been associated with oxidative stress and reduced neurogenesis in several brain regions which are implicated in depression including the hippocampus [25-27]. Therefore, the bidirectional relationship between psychological stress and physiological inflammatory responses may be a precipitating factor for depression and further inflammation in vulnerable individuals.

\section{Dysregulation of the Hypothalamic-Pituitary-Adrenal} Axis May Have Neurological Effects

The nervous system and skin interact via the actions of hypothalamic-pituitary-adrenal (HPA)-axis hormones and the upregulation of substance $P$ and calcitonin generelated peptide amongst others [28]. The HPA axis consists of hypothalamic corticotropin-releasing hormone (CRH) stimulating the release of pituitary adrenocorticotropic hormone (ACTH), which then stimulates the production of cortisol by the adrenal glands. Appropriate HPA axis reactivity to stressful stimuli is necessary to control immunological responses [29].
$\mathrm{CRH}$ has been shown to stimulate the local cutaneous production of cytokines and the keratinocyte expression of immune cell adhesion molecules [4]. Biopsies of psoriatic lesions show increased expression of $\mathrm{CRH}$, suggesting that it plays a role in the pathophysiology of the disease and possibly in skin-brain interactions [30]. TNF- $\alpha$ administration elevates plasma levels of ACTH and cortisol [31]. IL-6 can increase ACTH and cortisol, amplifying systemic pro-inflammatory circuits [32]. A chronic state of elevated levels of ACTH and cortisol can lead to glucocorticoid receptor insensitivity and an overall dysregulation of HPA pathways [33].

Interactively, psychological stress can increase the levels of cortisol and inflammatory cytokines [25, 34]. PS, $\mathrm{AD}$, and $\mathrm{HS}$ have been observed to worsen in response to psychological stress $[35,36]$. Patients with stress-reactive PS (PS that worsens during periods of stress) exhibit a blunted HPA axis response to acute social stressors as measured by serum cortisol levels [34]. Interestingly, it is social stress rather than cognitive or emotional stress that is discriminative in stress-reactive PS [34]. Hence, dysregulation of the HPA axis may contribute to inflammatory, physiological, and neurological effects.

\section{Evidence for an Association of Skin Disease with Cognitive Impairment}

It has been shown that PS patients may suffer from cognitive impairment [37-39]. For instance, a recent study on patients with severe PS revealed that they scored lower in neuropsychological tests that assessed working memory and executive function (e.g., the trail-making test, the Stroop color-word interference test, and the Beck Depression Inventory [BDI]) than a healthy control group and independently of depressive symptom severity [37]. In the same study, a longer duration of PS significantly correlated with worse outcomes on measures of working memory and executive function. This may be suggestive of the adverse effects of chronic inflammation on the prefrontal cortex which supports important cognitive processes such as decision-making and attention [37]. In a study on $\mathrm{AD}$ patients, cortisol, ACTH, and catecholamine concentrations were assessed before and after a stressful task (the Trier social stress test) to investigate the sensitivity of the HPA axis. The AD patients showed significantly attenuated cortisol and ACTH responses to stress [29].

HS is also a chronic, debilitating disease with a strong link to anxiety, depression, and substance abuse [40]. A cross-sectional study found that the individuals with HS $(n=94)$ reported significantly higher anxiety scores than the healthy controls, and that higher disease severity (Hur- 
ley stage III) was associated with higher anxiety levels [41]. In a recent study, the psychological trait of resilience, the ability to adapt well to adversity, moderated depression in HS, such that greater resilience was associated with lower depressive symptoms in HS patients [10]. Although, to the best of our knowledge, studies examining stress responses have not been conducted on HS patients, comorbidity with several endocrine disorders such as diabetes, acromegaly and Cushing's disease suggest that similar endocrine disruptions may play a role in HS [42].

\section{The Skin as a Source of Pro-Inflammatory Mediators}

An important driver of pathogenesis in many inflammatory skin disorders is the activation of cutaneous $T$ cells and dendritic cells and the upregulation of pro-inflammatory cytokines. Following migration to the skin and the release of pro-inflammatory cytokines, immune cells promote biological responses in keratinocytes which themselves are instigators of further inflammation [43]. Genome-wide comparative analyses report a high degree of genomic coincidence between PS and AD; however, opposing risk alleles and independent disease-specific loci within the epidermal differentiation complex and other regions have been identified that influence immune response [44].

It is now appreciated that each of these skin diseases, PS, AD, and HS, has a distinctive cytokine signature [45, 46]. PS is dominated by upregulation of the IL-17 axis, but with contributions from excess IL-12/23 and TNF- $\alpha$ [47]. IL-36 mutations have recently been discovered in some subtypes of PS [48]. Skin biopsies from AD sufferers show a predominance of T-helper-2-derived cytokines; it is suggested that this contributes to the mechanism of stress-induced inflammation in $\mathrm{AD}$ [29]. Recent effective targeted therapy directed at the IL-4/13 axis has demonstrated the importance of this pathway in AD [49]. The cytokine signature in HS is not fully elucidated, but may include dysregulation of TNF, IL-1, IL-12/23, IL-17, and IL-6 [50]. As discussed above, cytokines such as IL-6 and TNF- $\alpha$ may contribute to central nervous system changes and microglial remodeling [15].

\section{Evidence for Psychological Effects in Immune- \\ Modulating Interventional Clinical Trials for PS, AD, and HS}

Certain targeted therapeutic interventions directed at inflammatory skin diseases like PS, AD, and HS have investigated concurrent psychological effects. A phase 3 study of placebo or etanercept (50 mg twice weekly), a soluble TNF- $\alpha$ receptor, in patients with PS $(n=618)$ has

Inflammation and Depressive Comorbidity in Inflammatory Skin Disease indicated meaningful improvement in symptoms of depression as measured by the Hamilton Depression Rating Scale (HAM-D) and BDI at week 12 [51]. Patients with moderate-to-severe PS $(n=1,230)$ receiving $90 \mathrm{mg}$ of ustekinumab, a human IL-12/23 antibody, in a phase 3 clinical trial, showed significant improvement in symptoms of depression as measured by the Hospital Anxiety and Depression Scale (HADS) at week 12, when compared with placebo [52]. The use of adalimumab $(n=44$, $40 \mathrm{mg}$ every other week), a human monoclonal $\operatorname{IgG}_{1}$ antibody against TNF, versus placebo $(n=52)$, in moderateto-severe PS, resulted in a substantial reduction in depressive symptoms, as measured by the Zung Self-Rating Depression Scale (ZDS) after 12 weeks [53]. In 2 randomized placebo-controlled phase 3 studies of dupilumab, a monoclonal antibody that binds specifically to the shared $\alpha$-chain subunit of the IL- 4 and IL-13 receptors, symptoms of depression and anxiety (measured by HADS) were reduced significantly in patients with moderate and severe $\mathrm{AD}[54,55]$. The alleviation of pain by targeting inflammation in HS patients using adalimumab has also been shown to reduce depressive symptoms and pain scores [56].

\section{Opportunities for Interventions}

Figure 1 summarizes the potential relationship between skin-derived inflammatory mediators negatively impacting psychological well-being, and depression leading to increased skin inflammation, which creates a chronic exacerbating cycle. The interaction between psychological factors and inflammation suggest that interventions targeted at improving either aspect of the disease can help improve patients' quality of life. Indeed, interrupting any of these elements may help improve both skin inflammation and depressive psychological symptoms.

Importantly, studies on the effects of biologics (TNF or cytokine inhibitors) for patients with PS and AD report that these treatments are associated with significant reductions in depressive symptoms, as discussed above [3, 54]. Randomized controlled trials of the use of anti-inflammatory agents and cytokine inhibitors for the treatment of depression in nondermatological patients show similar results [57]. These observations demonstrate that pro-inflammatory cytokines specifically targeted by disease-modulating anti-cytokine therapies have a beneficial impact on psychological comorbidities such as depression. Physical metrics of disease severity, in conjunction with subjective patient-reported outcomes such as pain and depression, should therefore be a continued focus of therapeutic outcomes. In addition, evidence exists to 
show that anti-depressant treatment, particularly serotonin reuptake inhibitors, reduces levels of IL-6 and TNF- $\alpha$ [58]. However, the exact molecular mechanisms and signaling pathways underlying the association of depression with inflammatory skin disease remain incompletely understood.

Anti-inflammatory pharmacological agents targeting the skin have shown effectiveness in improving inflammatory symptoms, depressive symptoms, and overall quality of life in PS and AD. There is a scarcity of research into psychological outcomes following treatment with anti-inflammatory agents in $\mathrm{HS}$ and $\mathrm{AD}$, in addition to considering adjunctive psychological interventions in these patient populations.
In conclusion, the reviewed literature is supportive of an approach to management which accounts for the complex interaction between psychological and physiological factors in inflammatory conditions, with particular attention to improving the psychological burden of these disorders. The bidirectional relationship between psychological stress and physiological inflammatory responses may be a plausible conduit for the precipitation or exacerbation of depression and further cutaneous inflammation in vulnerable individuals.

\section{Disclosure Statement}

There were no conflicts of interest.

\section{References}

1 Dalgard FJ, Gieler U, Tomas-Aragones L, Lien L, Poot F, Jemec GBE, et al: The psychological burden of skin diseases: a cross-sectional multicenter study among dermatological out-patients in 13 European countries. J Invest Dermatol 2015;135:984-991.

2 Gupta MA, Gupta AK: Psychiatric and psychological co-morbidity in patients with dermatologic disorders: epidemiology and management. Am J Clin Dermatol 2003;4:833842.

3 Fleming P, Roubille C, Richer V, Starnino T, McCourt C, McFarlane A, et al: Effect of biologics on depressive symptoms in patients with psoriasis: a systematic review. J Eur Acad Dermatol Venereol 2014;29:1063-1070.

4 Connor CJ, Liu V, Fiedorowicz JG: Exploring the physiological link between psoriasis and mood disorders. Dermatol Res Pract 2015; 2015:1-11.

5 Janse IC, Deckers IE, van der Maten AD, Evers AW, Boer J, van der Zee HH, et al: Sexual health and quality of life are impaired in hidradenitis suppurativa: a multicenter crosssectional study. Br J Dermatol 2017;176: 1042-1047.

6 Gooderham N: Atopic dermatitis, depression, and suicidality. J Cutan Med Surg 2017;21: 237-242.

7 Rapp SR, Feldman SR, Exum ML, Fleischer $\mathrm{AB}$, Reboussin DM: Psoriasis causes as much disability as other major medical diseases: J Am Acad Dermatol 1999;41:401-407.

8 Vazquez BG, Alikhan A, Weaver AL, Wetter DA, Davis MD: Incidence of hidradenitis suppurativa and associated factors: a populationbased study of Olmsted County, Minnesota. J Invest Dermatol 2013;133:97-103.

9 Shavit E, Dreiher J, Freud T, Halevy S, Vinker S, Cohen AD: Psychiatric comorbidities in 3207 patients with hidradenitis suppurativa. J Eur Acad Dermatol Venereol 2015;29:371-376.
10 Kirby JS, Butt M, Esmann S, Jemec GBE: Association of resilience with depression and health-related quality of life for patients with hidradenitis suppurativa. JAMA Dermatol 2017;153:1263-1269.

11 Nicholas MN, Gooderham MJ: Atopic dermatitis, depression, and suicidality. J Cutan Med Surg 2017;21:237-242.

12 Thorlacius L, Cohen AD, Gislason GH, Jemec GBE, Egeberg A: increased suicide risk in patients with hidradenitis suppurativa. J Invest Dermatol 2018;138:52-57.

13 Chi CC, Chen TH, Wang SH, Tung TH: Risk of suicidality in people with psoriasis: a systematic review and meta-analysis of cohort studies. Am J Clin Dermatol 2017;18:621627.

14 Huilaja L, Tiri H, Jokelainen J, Timonen M, Tasanen K: Patients with hidradenitis suppurativa have a high psychiatric disease burden: a Finnish nationwide registry study. J Invest Dermatol 2018;138:46-51.

15 Dowlati Y, Herrmann N, Swardfager W, Liu H, Sham L, Reim EK, Lanctot KL: A metaanalysis of cytokines in major depression. Biol Psychiatry 2010;67:446-457.

16 Rosenblat JD, Cha DS, Mansur RB, McIntyre RS: Inflamed moods: a review of the interactions between inflammation and mood disorders. Prog Neuropsychopharmacol Biol Psychiatry 2014;53:23-34.

17 Valentine AD, Meyers CA, Kling MA, Richelson E, Hauser P: Mood and cognitive side effects of interferon-alpha therapy. Semin Oncol 1998;25:39-47.

18 Capuron L, Raison CL, Musselman DL, Lawson DH, Nemeroff CB, Miller AH: Association of exaggerated HPA axis response to the initial injection of interferon-alpha with development of depression during interferonalpha therapy. Am J Psychiatry 2003;160: 1342-1345.
19 Setiawan E, Wilson AA, Mizrahi R, Rusjan PM, Miler L, Rajkowska G, et al: Role of translocator protein density, a marker of neuroinflammation, in the brain during major depressive episodes. JAMA Psychiatry 2015;72: 268-275.

20 Reichenberg A, Yirmiya R, Schuld A, Kraus T, Haack M, Morag A, Pollmacher T: Cytokineassociated emotional and cognitive disturbances in humans. Arch Gen Psychiatry 2001; 58:445-452.

21 Hodes GE, Pfau ML, Leboeuf M, Golden SA, Christoffel DJ, Bregman D, et al: Individual differences in the peripheral immune system promote resilience versus susceptibility to social stress. Proc Natl Acad Sci USA 2014;111: 16136-16141.

22 Yirmiya R, Rimmerman N, Reshef R: Depression as a microglial disease. Trends Neurosci 2015;38:637-658.

23 Miller AH, Maletic V, Raison CL: Inflammation and its discontents: the role of cytokines in the pathophysiology of major depression. Biol Psychiatry 2017;65:1-10.

24 Dantzer R, O'Connor JC, Lawson MA, Kelley KW: Inflammation-associated depression: from serotonin to kynurenine. Psychoneuroendocrinology 2011;36:426-436.

25 Dantzer R, O'Connor JC, Freund GG, Johnson RW, Kelley KW: From inflammation to sickness and depression: when the immune system subjugates the brain. Nat Rev Neurosci 2008;9:46-56.

26 Sapolsky RM: Stress and the brain: individual variability and the inverted-U. Nat Neurosci 2015; 18:1344-1346.

27 Lee AL, Ogle WO, Sapolsky RM: Stress and depression: possible links to neuron death in the hippocampus. Bipolar Disord 2002;4: 117-128. 
28 Sartorius K, Emtestam L, Lapins J, Johansson O: Cutaneous PGP 9.5 distribution patterns in hidradenitis suppurativa. Arch Dermatol Res 2010;302:461-468.

29 Buske-Kirschbaum A, Geiben A, Höllig H, Morschhäuser E, Hellhammer D: Altered responsiveness of the hypothalamus-pituitaryadrenal axis and the sympathetic adrenomedullary system to stress in patients with atopic dermatitis. J Clin Endocrinol Metabol 2002; 87:4245-4251.

30 Quevedo ME, Slominski A, Pinto W, Wei E, Wortsman J: Pleiotropic effects of corticotropin releasing hormone on normal human skin keratinocytes. In Vitro Cell Dev Biol Anim 2001;37:50-54

31 Raison CL, Miller AH: Is depression an inflammatory disorder? Curr Psychiatry Rep 2011;13:467-475

32 Fonseca JE, Santos MJ, Canhao H, Choy E: Interleukin-6 as a key player in systemic inflammation and joint destruction. Autoimmun Rev 2009;8:538-542.

33 Krishnadas R, Cavanagh J: Depression: an inflammatory illness? J Neurol Neurosurg Psychiatry 2012;83:495-502.

34 Richards HL, Ray DW, Kirby B, Mason D, Plant D, Main CJ, Fortune DG, Griffiths CE Response of the hypothalamic-pituitary-adrenal axis to psychological stress in patients with psoriasis. Br J Dermatol 2005;153:11141120.

35 Picardi A, Abeni D, Melchi CF, Puddu P, Pasquini P: Psychiatric morbidity in dermatological outpatients: an issue to be recognized. $\mathrm{Br}$ J Dermatol 2000;143:983-991.

36 Von der Werth J, Jemec G: Morbidity in patients with hidradenitis suppurativa. Br J Dermatol 2001;144:809-813.

37 Marek-Jozefowicz L, Jaracz M, Placek W, Czajkowski R, Borkowska A: Cognitive impairment in patients with severe psoriasis. Postepy Dermatol Alergol 2017;34:120-125.

38 Gisondi P, Sala F, Alessandrini F, Avesani V, Zoccatelli G, Beltramello A, et al: Mild cognitive impairment in patients with moderate to severe chronic plaque psoriasis. Dermatology 2014;228:78-85.
39 Colgecen E, Celikbilek A, Keskin DT: Cognitive impairment in patients with psoriasis: a cross-sectional study using the Montreal Cognitive Assessment. Am J Clin Dermatol 2016;17:413-419.

40 Shlyankevich J, Chen AJ, Kim GE, Kimball AB: Hidradenitis suppurativa is a systemic disease with substantial comorbidity burden: a chart-verified case-control analysis. J Am Acad Dermatol 2014;71:1144-1150.

41 Kouris A, Platsidaki E, Christodoulou C, Efstathiou V, Dessinioti C, Tzanetakou V, et al: Quality of life and psychosocial implications in patients with hidradenitis suppurativa. Dermatology 2016;232:687-691.

42 Fimmel S, Zouboulis CC: Comorbidities of hidradenitis suppurativa (acne inversa). Dermatoendocrinology 2010;2:9-16.

43 Nestle FO, Di Meglio P, Qin J-Z, Nickoloff BJ: Skin immune sentinels in health and disease. Nat Rev Immunol 2009;9:679-691.

44 Baurecht $\mathrm{H}$, Hotze $\mathrm{M}$, Brand S, Buning C, Cormican P, Corvin A, et al: Genome-wide comparative analysis of atopic dermatitis and psoriasis gives insight into opposing genetic mechanisms. Am J Hum Genet 2015;96:104120.

45 Werfel T, Allam JP, Biedermann T, Eyerich K, Gilles S, Guttman-Yassky E, et al: Cellular and molecular immunologic mechanisms in patients with atopic dermatitis. J Allergy Clin Immunol 2016;138:336-349.

46 Schett G, Elewaut D, McInnes IB, Dayer JM, Neurath MF: How cytokine networks fuel inflammation: toward a cytokine-based disease taxonomy. Nat Med 2013;19:822-824.

47 Martin DA, Towne JE, Kricorian G, Klekotka P, Gudjonsson JE, Krueger JG, Russell CB: The emerging role of IL-17 in the pathogenesis of psoriasis: preclinical and clinical findings. J Invest Dermatol 2013;133:17-26.

48 Towne JE, Sims JE: IL-36 in psoriasis. Curr Opin Pharmacol 2012;12:486-490.

49 Matsunaga MC, Yamauchi PS: IL-4 and IL-13 inhibition in atopic dermatitis. J Drugs Dermatol 2016;15:925-929.

50 Hoffman LK, Ghias MH, Garg A, Hamzavi $\mathrm{IH}$, Alavi A, Lowes MA: Major gaps in understanding and treatment of hidradenitis suppurativa. Semin Cutan Med Surg 2017;36: 86-92.
51 Tyring S, Gottlieb A, Papp K, Gordon K, Leonardi C, Wang A, et al: Etanercept and clinical outcomes, fatigue, and depression in psoriasis: double-blind placebo-controlled randomised phase III trial. Lancet 2006;367: 29-35.

52 Langley RG, Feldman SR, Han C, Schenkel B, Szapary P, Hsu MC, et al: Ustekinumab significantly improves symptoms of anxiety, depression, and skin-related quality of life in patients with moderate-to-severe psoriasis: results from a randomized, double-blind, placebo-controlled phase III trial. J Am Acad Dermatol 2010;63:457-465.

53 Menter A, Augustin M, Signorovitch J, Yu AP, Wu EQ, Gupta SR, Bao Y, Mulani P: The effect of adalimumab on reducing depression symptoms in patients with moderate to severe psoriasis: a randomized clinical trial. J Am Acad Dermatol 2010;62:812-818.

54 Simpson EL, Bieber T, Guttman-Yassky E, Beck LA, Blauvelt A, Cork MJ, et al: Two phase 3 trials of dupilumab versus placebo in atopic dermatitis. N Engl J Med 2016;375: 2335-2348.

55 Simpson EL, Gadkari A, Worm M, Soong W, Blauvelt A, Eckert L, et al: Dupilumab therapy provides clinically meaningful improvement in patient-reported outcomes (PROs): A phase IIb, randomized, placebo-controlled, clinical trial in adult patients with moderate to severe atopic dermatitis (AD). J Am Acad Dermatol 2016;75:506-515.

56 Scheinfeld N, Sundaram M, Teixeira H, Gu Y, Okun M: Reduction in pain scores and improvement in depressive symptoms in patients with hidradenitis suppurativa treated with adalimumab in a phase 2 , randomized, placebo-controlled trial. Dermatol Online J 2016;22:pii: 13030/qt38x5922j.

57 Köhler O, Benros ME, Nordentoft M, Farkouh ME, Iyengar RL, Mors O, Krogh J: Effect of anti-inflammatory treatment on depression, depressive symptoms, and adverse effects. JAMA Psychiatry 2014;71:1381-1311.

58 Hannestad J, DellaGioia N, Bloch M: The effect of antidepressant medication treatment on serum levels of inflammatory cytokines: a meta-analysis. Neuropsychopharmacology 2011;36:2452-2459. 